\title{
HARECES Measurements of Carbon K Shell Excitation in Graphite
}

Nestor J. Zaluzec*, Mark G. Blackford**, Katherine L. Smith** and Michael Colella

*Electron Microscopy Center, Argonne National Laboratory, Argonne, IL, USA 60439

**Materials Science and Engineering, Australian Nuclear Science and Technology Organization, Menai, New South Wales,2234 Australia.

High Angular Resolution Electron Channeling Electron Spectroscopy (HARECES) is an analogue of the corresponding X-ray technique (HARECXS) in which one measures the variation of a spectroscopic signal with illumination angle [1-2]. Todate this methodology has been a rarely employed technique in EELS due to the intricacies of instrumenting the experiment and the weak signals which are obtained. We have implemented a computationally mediated procedure to achieve these measurements on both a JEOL 2010F and FEI Tecnai F20 TEM/STEM instruments and in this work report on preliminary measurements of the angular distribution of Carbon K Shell excitation which permits comparison to previous work by Leapman etal [3].

The sample chozen for this study was a large grain crystal specimen of pyrolytic graphite produced by cleavage along the basal plane. The sample was mounted on a $3 \mathrm{~mm}$ diameter copper grid, plasma cleaned and then introduced into the respective instruments. The measurements reported herein were carried out in a JEOL 2010F at ANSTO operating at $200 \mathrm{kV}$ and were acquired with the incident probe in nearly parallel illumination conditions $(\alpha<0.05 \mathrm{mR})$ achieved by adjusting the incident beam convergence using the $2010 \mathrm{~F}$ alpha selector and $\mathrm{C} 2$ lens excitation. The angular resolution of the measured signal was controlled by a combination of camera length and a fixed 0.6 $\mathrm{mm}$ diameter aperture at the entrance to the electron spectrometer and was $<0.1 \mathrm{mR}$ for the data presented. The incident beam was digitally tilted through $\pm 1.5 \mathrm{~g}$ where $\mathrm{g}=\langle 1-100\rangle$ in digital steps using a custom built external computer control interface which routed the digital scan generator signal from an Emispec ESVision data acquisition system to the STEM scan unit of the microscope and then into the hollow cone illumination device HCID10. Electron Energy Loss spectra where recorded at $0.3 \mathrm{eV} / \mathrm{chn}$ over 1024 Channels using a Gatan Model 2001GIF, whose data was transferred to the Emispec system during acquisition and subsequently analyzed on a Mac OS X computer using computer programs written by the authors. Figure 1 presents a partial view of experimental dispersion surface measured for the Carbon $\mathrm{K}$ shell just off the [0001] basal plane along g. Figure 2 presents the corresponding K Shell line profiles for different angular integration windows of $0.26,1.8$ and $35 \mathrm{mR}$ which were obtained by digitally integration of the data in Fig 1. These results compare very well with work of Leapman etal even though tilting the illumination instead of the post specimen deflection carried out the measurements. Calculations are in progress to compare the results of density functional theory electronic structure calculations using WEIN 2K [4] and this high angular resolution data.

References

[1] Zaluzec N.J. Microscopy Microanalysis Vol 6, Sup 2, 938 (1999).

[2] Cowley J. , Acta Crys. 1733 (1964)

[3] Leapman R.D etal , Phys Rev B, Vol 28258 (1983).

[4] WEIN2K see http://www.wien2k.at/

[5] This work was supported in part by the U.S. DoE under BES MS W-31-109-Eng-38 at ANL NJZ gratefully acknowledges travel support by ANSTO which facilitated some of this work. 


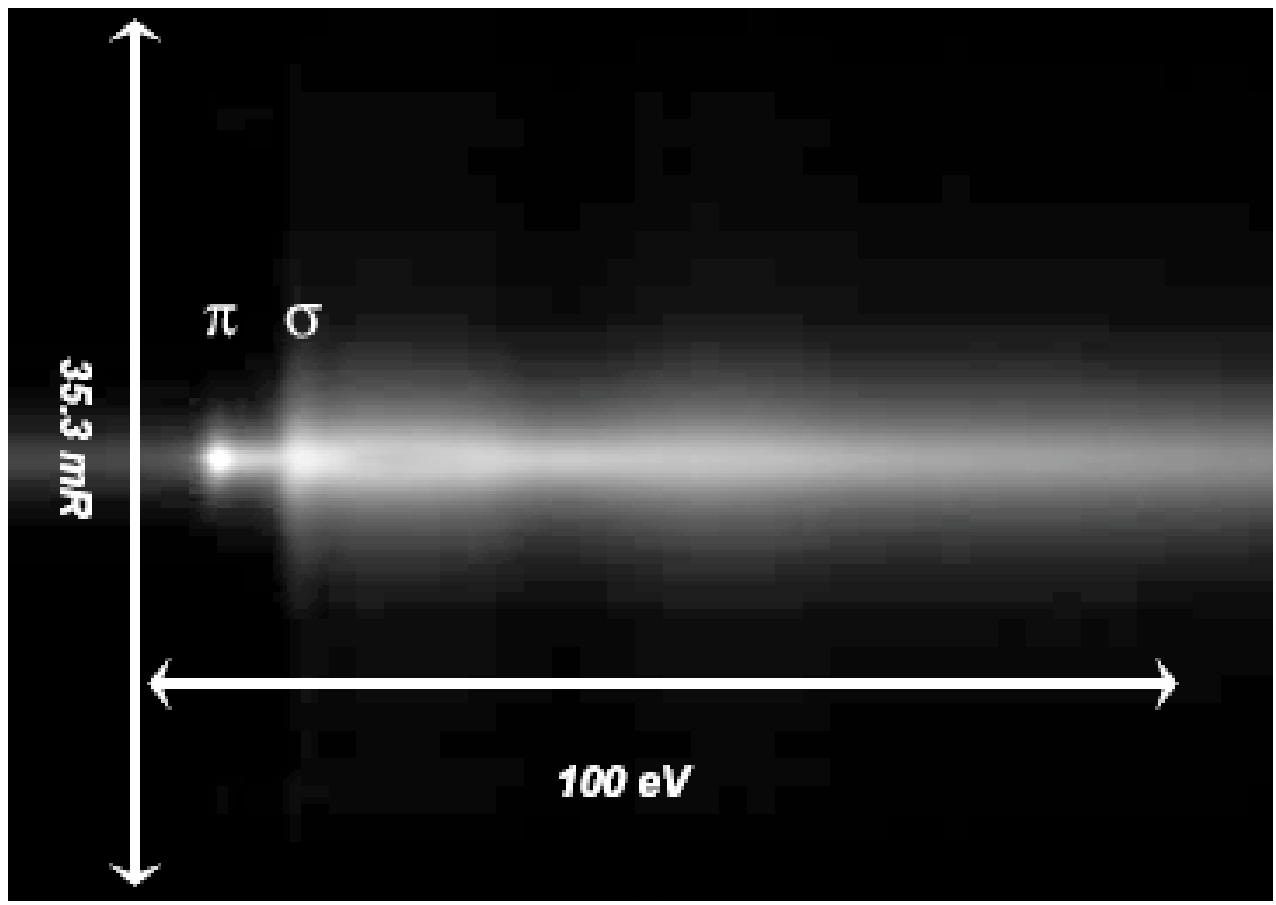

Figure 1. HARECES Measurements of the C Kshell in Graphite along $<1-1 \quad 0 \quad 0>$ note the intensity of the $<1-100>$ reflections are too weak to view in this image but are discernable on contrast expansion. The Graphite $\pi$ and $\sigma$ states are indicated.

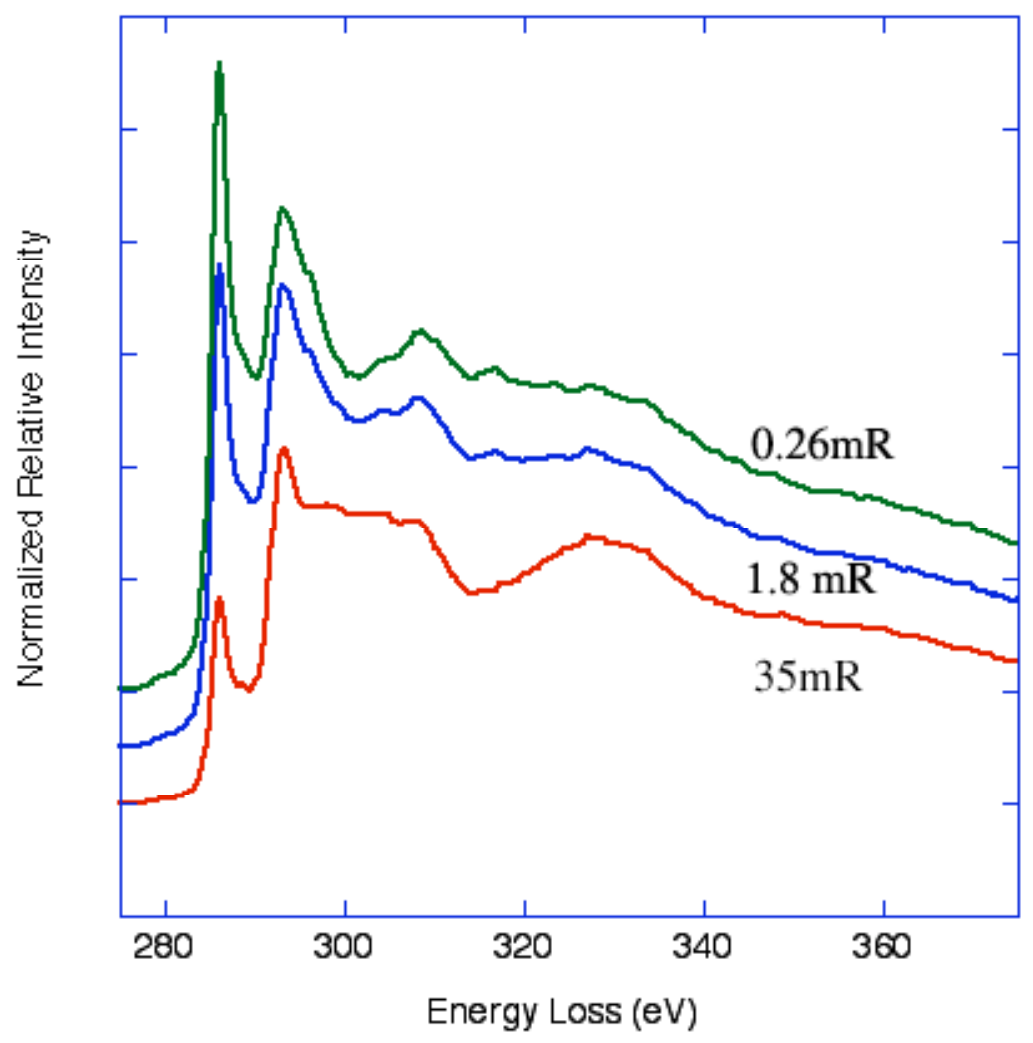

Figure 2. Angular integration of the HARECES data of Figure 1 for effective collection angles of 0.26 (green/top), 1.8 (blue/middle) and 35 (red/bottom) mR. These data demonstrate the localization of $\pi$ and $\sigma$ states on the K shell. 